\title{
On the magnetic structure of the solar transition region
}

\author{
Philip Judge and Rebecca Centeno \\ High Altitude Observatory, National Center for Atmospheric Research ${ }^{1}$, P.O. Box 3000, \\ Boulder CO 80307-3000, USA
}

\begin{abstract}
We examine the hypothesis that "cool loops" dominate emission from solar transition region plasma below temperatures of $2 \times 10^{5} \mathrm{~K}$. We compare published VAULT images of $\mathrm{H} \mathrm{L} \alpha$, a lower transition region line, with nearcontemporaneous magnetograms from Kitt Peak, obtained during the second flight (VAULT-2) on 14 June 2002. The measured surface fields and potential extrapolations suggest that there are too few short loops, and that L $\alpha$ emission is associated with the base regions of longer, coronal loops. VAULT-2 data of network boundaries have an asymmetry on scales larger than supergranules, also indicating an association with long loops. We complement the Kitt Peak data with very sensitive vector polarimetric data from the Spectro-Polarimeter on board Hinode, to determine the influence of very small magnetic concentrations on our analysis. From these data two classes of behavior are found: within the cores of strong magnetic flux concentrations $\left(>5 \times 10^{18} \mathrm{Mx}\right)$ associated with active network and plage, small-scale mixed fields are absent and any short loops can connect just the peripheries of the flux to cell interiors. Core fields return to the surface via longer, most likely coronal, loops. In weaker concentrations, short loops can connect between concentrations and produce mixed fields within network boundaries as suggested by Dowdy and colleagues. The VAULT-2 data which we examined are associated with strong concentrations. We conclude that the cool loop model applies only to a small fraction of the VAULT-2 emission, but we cannot discount a significant role for cool loops in quieter regions. We suggest a physical picture for how network L $\alpha$ emission may occur through the cross-field diffusion of neutral atoms from chromospheric into coronal plasma.
\end{abstract}

Subject headings: Sun: atmosphere - Sun: chromosphere - Sun: transition region - Sun: corona - Sun: magnetic fields 


\section{Introduction}

In spite of a century or so of research, the solar atmosphere continues to challenge our understanding. As well as the well-known problems of identifying the causes of coronal and chromospheric heating, other phenomena such as spicules, flares, explosive events, and atmospheric dynamics in general, remain only partly understood.

A particularly stubborn puzzle relates to the structure of the solar transition region (henceforth "TR") - plasma between the chromosphere and corona. The chromosphere and TR are particularly bright over magnetic field concentrations. Outside of sunspots, the concentrations form the supergranular network boundaries (henceforth, "NB"s). Cell interiors ("CI"s) are less bright. The supergranular network pattern eventually disappears at coronal temperatures $T_{e} \gtrsim 10^{6} \mathrm{~K}$ (Tousey 1971; Reeves 1976). The upper TR (where the electron temperature $T_{e}$ lies between say $2 \times 10^{5} \mathrm{~K}$ and coronal temperatures of $10^{6} \mathrm{~K}$ ) is adequately described by magnetic field-aligned thermal conduction down from the corona (Gabriel 1976; Jordan 1980), but that the lower TR $\left(10^{4} \mathrm{~K}<T_{e}<2 \times 10^{5} \mathrm{~K}\right)$ is not so easily understood (see, for example, the reviews by Mariska 1992; Anderson-Huang 1998). Models dominated by heat conduction along magnetic field lines fail to produce enough bright emission from the lower transition by orders of magnitude. In other words, the differential emission measure in the lower transition region is far higher than predicted by such models. Two points argue, however, in favor of conduction as a source of energy for the lower TR: First, the conductive flux is of the right order of magnitude to explain the radiation losses there. Second, the relative intensities of coronal and TR lines vary surprisingly little over the solar surface (i.e., the differential emission measure has a universal shape), suggesting an energetic link.

Attempts to fix the problem have either largely failed, or raised questions of an equally troubling nature. For example, models based on heat flow parallel to magnetic field lines were explored which dropped earlier restrictions on magnetic field geometry and a static picture. But such calculations fail to account for the brightness of the lower transition region (Gabriel 1976, Pneuman and Kopp 1978, Athay 1981, Woods 1986). A variation on such models was developed by Cally (1990), introducing enhanced heat fluxes using transport by turbulent eddies. Cally found that the mixing length (a free parameter) should scale as $T_{e}^{-1.5}$ to account for observations. But this is somewhat unsatisfactory because there is no physical reason why the turbulent transport should behave like this. Ashbourn and Woods (2001) presented a more promising model in which the ion-acoustic instability sets in owing to the high electron drift speed associated with heat conduction, in the middle TR. The instability makes the perpendicular ion thermal conductivity large and dominant below $10^{5} \mathrm{~K}$, thereby providing a lower temperature gradient and higher emission measure 
than fluid approximations give. The downward heat flux was also explored by Fontenla et al. (1990,1991,1993,2002; henceforth "FAL") in multi-fluid models where diffusion and bulk flows transport cool material to hot regions, thereby extracting and radiating much of the available energy in the corona.

The essential property of all such models is that they produce a geometrically thin lower TR, where emission is confined to a layer just a few tens of $\mathrm{km}$ deep. As discussed in a series of papers by Feldman and colleagues (e.g., Feldman 1983), UV, EUV and X-ray images present serious challenges for such thin models. Such observations have prompted others to set aside conduction, instead focusing on local sources of heat within the TR, such as Joule dissipation (e.g., Rabin and Moore 1984, Roumeliotis 1991) or parameterized forms of heat dissipation (e.g. Antiochos and Noci 1986; Patsourakos et al. 2007). Such models are similar to chromospheric models (e.g. Vernazza et al. 1981), in the sense that local heating is balanced by radiation losses ${ }^{1}$. In such models, conduction plays no role, and instead the heights of the structures or "cool loops" are physically limited by the pressure scale height, the temperature scale heights being larger (Antiochos and Noci 1986). Even in loops near $10^{5} \mathrm{~K}$, the height cannot exceed $5 \mathrm{Mm}$ for equilibrium to exist, for heating functions which are not of a special form (Cally and Robb 1991). Limb observations of active regions reveal cool material much higher, but such structures are almost always dynamic (e.g. Di Giorgio et al. 2003).

Dowdy et al. (1986) pointed out that one would expect magnetic field of mixed polarity within the supergranular NBs, a feature absent from the model of (Gabriel 1976). Dowdy and colleagues suggested TR emission below $\sim 10^{5} \mathrm{~K}$ is dominated by radiation from locally heated loops which connect such opposite polarity photospheric fields, on scales of $\lesssim 10 \mathrm{Mm}$. Their picture is illustrated by figure 1, taken from their 1986 article.

Sánchez Almeida et al. (2007) searched for magnetic signatures of footpoints of cool loops, using data from the SUMER (Wilhelm et al. 1995) and MDI (Scherrer et al. 1995) instruments on the SOHO spacecraft, and $\mathrm{G}$ band bright point data from the Dutch Open Telescope (Hammerschlag and Bettonvil 1998). Their analysis was inconclusive, because, as we will find, neither SUMER nor MDI (full-disk) data have sufficient spatial resolution (1".5 and $4^{\prime \prime}$ respectively), or magnetic sensitivity.

The present paper studies the proposal of Dowdy et al. by examining very high an-

\footnotetext{
${ }^{1}$ However, unlike the chromosphere, at transition region temperatures the plasma is almost fully ionized and there is no internal heat sink (latent heat of ionization) which acts, in the chromosphere, as a thermostat. Locally heated and cooled models of the TR are therefore susceptible to thermal instability (Cally and Robb 1991).
} 
gular resolution $\left(\approx 0^{\prime \prime} .3\right)$ images of the lower transition from the VAULT instrument (Korendyke et al. 2001), and comparing these with magnetic fields and extrapolations from nearly-simultaneous magnetogram data. Since the magnetic data available do not have the sensitivity of recent data, we also examine spectroheliograms of the Sun from the spectropolarimeter (SP, Lites et al. 2001) on the Hinode spacecraft (Kosugi et al. 2007). We will argue that several observed properties of the VAULT L $\alpha$ emission are not compatible with the cool loop hypothesis.

\section{UV data of the cool transition region and corona}

We have re-analyzed data described by Patsourakos et al. (2007), augmented by UV and EUV data from SOHO and TRACE (Handy et al. 1999). The VAULT data were obtained during the second instrument flight (VAULT-2) on 14 June 2002 near 18:12 UT. The field of view is $375^{\prime \prime} \times 257^{\prime \prime}$ centered near coordinates $\left(-600^{\prime \prime},+260^{\prime \prime}\right)$ relative to solar disk center. The instrument captured both active plage areas south and east of the center of the FOV, and the quieter areas to the NW. The pixel size is $0{ }^{\prime} .125$ and resolution $\approx 0$. 3 . Figure 2 shows contextual images of the photosphere (MDI instrument) and corona (EIT, Delaboudiniere et al. 1995) from the SOHO spacecraft, together with a VAULT-2 image ${ }^{2}$.

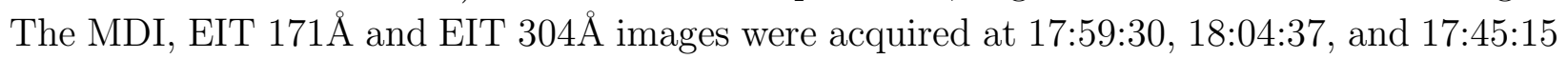
UT respectively. The VAULT-2 image was coaligned with the the EIT He II $304 \AA$ image, crudely by eye. It was found to be centered near $\left(-612^{\prime \prime}, 260^{\prime \prime}\right)$, and rotated clockwise by $9^{\circ}$. Furthermore the pixel sizes were found to be $0^{\prime \prime} .125$ and $0^{\prime \prime} .115$ in $x$ and $y$ respectively. (The different pixel scales found are not in disagreement with measurements by the VAULT team, C. Korendyke, private communication 2008). This co-alignment is reliable only to within $\approx \pm 2-3^{\prime \prime}$, but is sufficiently accurate for our purposes.

Focusing on the "quiet" NW sector of the images, the brightest L $\alpha$ emission occurs over fairly strong, unipolar magnetic flux concentrations (see section 3). A feature of the data receiving much attention (see Patsourakos et al. 2007, in particular their fig. 2) is the fine thread-like structure. The threads are most obvious seen against the darker CIs in the neighborhood of the bright concentrations. Similar fine structure has been seen for many years in the related, but notoriously difficult to interpret, $\mathrm{H} \alpha$ line (e..g. Kiepenheuer 1953; Martin et al. 1994). The chromospheric network in L $\alpha$ contains many such threads, combining to form a collective network emission of order 10" width (Patsourakos et al. 2007, their fig. 2). These authors showed that the fine structure is missing from data with lower

\footnotetext{
${ }^{2}$ The image analyzed is img04.png from http://wwwsolar.nrl.navy.mil/rockets/vault/archives.html.
} 
angular resolution than $\approx 1^{\prime \prime}$. In their picture, much of the emission from each concentration originates from the threads, which are assumed to be small loops.

Patsourakos et al. (2007) also noted that "The threads are, at a first approximation, radially distributed or slightly bent around the cell centers, which suggests that they could correspond to closed structures, i.e. small loops with temperatures in the temperature formation range of Ly $\alpha . "$ However, such patterns are not actually common in the VAULT-2 data analyzed by them. Inspection of Fig. 2 of Patsourakos et al. (2007), an area of $100^{\prime \prime} \times 60^{\prime \prime}$ containing say 4 or so supergranular cells, shows that the threads are all oriented between $\approx-40^{\circ}$ and $10^{\circ}$ of the direction of the $y$ axis (see also figure 3 ). Thus the thread orientations are organized on supergranular scales (i.e. much larger than granules), and larger still near active regions. The bright $\mathrm{L} \alpha$ patches appear like small "comets" whose tails are comprised of the threads, the "heads" of the comets pointing generally towards the active region in the SE of the image (bottom left of the figure). Such thread orientations are at odds with magnetic loop footpoints which might be expected from more random granular and supergranular forcing of surface magnetic fields. The "comets" are reminiscent of patterns seen in $\mathrm{H} \alpha$ data, particularly in the neighborhood of filaments, for many years (Martin et al. 1994; Low 1996).

Figure 3 shows the VAULT-2 data (yellow) together with TRACE $171 \AA$ data (four summed images) of the lower corona (blue), obtained close to 17:45 UT. TRACE data were aligned to VAULT-2 data using the EIT co-alignment. While $171 \AA$ coronal emission is present almost everywhere, it is brightest only in the vicinity of the plage and filament. White regions in the image shows areas where both features are bright, including some "moss" emission (slightly granulated structure) in the neighborhood of the filament, but also including some loops slightly further from the filament region. Use of a different color table shows coronal moss emission throughout the plage, with weak 171 emission even in the "quiet" region (upper right corner). $171 \AA$ moss is associated with conductive heating from overlying hotter loops (Fletcher and de Pontieu 1999; Berger et al. 1999). The coronal emission will be related to magnetic field extrapolations below.

VAULT images from an earlier flight (VAULT-1) were analyzed by Korendyke et al. (2001); Vourlidas et al. (2001). These images contain qualitatively similar features to those seen in the data studied here, including moss and threads organized into comet-like patterns in the region within $\approx 100^{\prime \prime}$ of the filament. The VAULT-1 DATA contain more quiet areas which contain network concentrations hosting significant, but less ordered, thread emission (fig. 5 of Korendyke et al. 2001).

To summarize, the essential features of the VAULT-2 data under scrutiny are: 
- Much of the emission is organized into thin threads, originating in the network cell boundaries, most visible against the dark cell interiors,

- near large-scale coronal structures and organized photospheric magnetic fields (active regions), the threads have characteristic orientations on scales in excess of supergranules,

- within plage or large active network boundaries, the emission appears to have a small scale granular structure associated with conductively heated coronal emission called "moss" (Vourlidas et al. 2001).

\section{Magnetic fields measured at the epoch of the VAULT-2 observations}

MDI full-disk data have a $1 \sigma$ noise level of $17 \mathrm{Mx} \mathrm{cm}^{-2}$ per $1^{\prime \prime} .984 \times 1^{\prime \prime} .984$ pixel, determined from the spatial power spectrum assuming the noise is "white". Fortunately, higher quality longitudinal magnetograms from the Kitt Peak Vacuum Telescope synoptic program are available, here we analyze a scan taken between 16:00 and 16:55 UT on June 142002. These data have pixels of $1^{\prime \prime} .148 \times 1^{\prime \prime}$. 148 , each with a noise of $\approx 2.8 \mathrm{Mx} \mathrm{cm}^{-2}$. Table 1 lists the relative sensitivities of these and other magnetograms. The KPVT instrument can detect the ubiquitous "salt and pepper" weak longitudinal field, with fluxes of $(65 \rightarrow 100) \times 10^{15}$ Mx, discovered many years ago by Livingston and Harvey (1971). Such small fluxes are invisible to MDI.

We aligned the KPVT data with VAULT-2 by eye, using the unsigned magnetic flux, we estimate the accuracy of the alignment is no worse than $3^{\prime \prime}$, probably better. This apparently large uncertainty arises because although there is a strong correlation between chromospheric and TR emission and absolute magnetic flux, the correlation is only on scales in excess of a few seconds of arc (Skumanich et al. 1975; Sánchez Almeida et al. 2007). Variations with time and small scale spatial variations limit the co-alignment accuracy. We rotated both datasets to disk center assuming that $\mathrm{L} \alpha$ and the KPNO data arise from the same spherical surface. This is manifestly incorrect, given that $\mathrm{L} \alpha$ is formed at least $2 \mathrm{Mm}$ above the photosphere and has contributions from spicules extending to $10 \mathrm{Mm}$ or so. Thus after the rotation we re-did the co-alignment. Lastly, we assumed that the observed fields are statistically radial, and re-computed the line-of-sight field on the rotated surface. (Our comparisons of field line morphology with $\mathrm{L} \alpha$ images are insensitive to the exact choice of radial vs. vertical field, and our calculations are only potential anyway).

We computed the potential magnetic field from this surface, assumed to be flat, using 
the Fourier method ${ }^{3}$. These calculations crudely indicate the morphology of magnetic fields overlying the surface fields, assuming that sources (i.e. currents) of magnetic field are negligible except those sub-surface currents responsible for the surface fields. Figure 4 shows VAULT data, contours of magnetic flux density, and field lines superposed. The field lines were plotted only if both footpoints exceeded twice the noise level of $2.8 \mathrm{Mx} \mathrm{cm}{ }^{-2}$, but the extrapolations themselves include all pixels. In this way we reject more than $95 \%$ of field lines which arise solely from noise. (Figure 5 below includes all field lines regardless of signalto-noise ratios, to illustrate that rejection of noisy data is not a critical issue). The plotted field lines were evenly sampled to avoid overcrowding here and in later figures. The region observed by VAULT is dominated by flux of positive polarity. Both short and long loops are aligned locally in generally the same direction. The large positive flux region connects to negative polarity fields in the SW corner and outside of the plotted field of view. The overwhelming amount of positive flux guides essentially all field lines away from it, in the potential fields shown.

Let us consider the extrapolated field lines as possible plasma loops, and first examine those of length $\leq 10 \mathrm{Mm}$. (Lengths and heights of potential field loops are on average related, but we also discriminate between low and high lying loops in the following figures). Such short loops are candidates for the "cool loop" model of $\mathrm{L} \alpha$ emission. If the emission were dominated by such structures, we would expect to find a correlation between bright thread emission and the position and density of these field lines. There are indeed places where this is the case, near $x, y=70,150 \mathrm{Mm}$ and $x, y=140,110 \mathrm{Mm}$ in the figure, for example, but in most cases the bright emission and short loop densities are poorly correlated. Figure 5 shows a close up of flux concentrations ${ }^{4}$ centered near $x, y=-500^{\prime \prime},+290^{\prime \prime}$ in figure 2 , showing many more short loops and their properties compared to the $\mathrm{L} \alpha$ threads. In this case we set no limits on the footpoint signal-to-noise ratios in selecting field lines, to see if excluding noisy pixels might introduce an important bias. It does not, it simply reduces the number of field lines with at least one footpoint in the CI regions. Thus, in figure 5, one finds areas where cool loops are indeed plausible (e.g. between $x, y=15,60^{\prime \prime}$, $\mathrm{a}^{\prime \prime}$ nd $x, y=55,60^{\prime \prime}$,)", but there are other areas where short loops are found without L $\alpha$ threads (most other locations in the figure). In any case, these short loops tend to connect only the peripheries of flux

\footnotetext{
${ }^{3}$ In all figures except 6 and 7, a larger field of view was used to compute the potential fields than is plotted, to try to avoid periodic artifacts.

${ }^{4}$ We refine our definition of "concentration" here to refer to an aggregation of flux of one polarity which defines part of a NB. Typically these have an area of $\gtrsim 1 \mathrm{Mm}^{2}$ and a flux $\gtrsim 10^{18} \mathrm{Mx}$, about three times the detection limit of MDI full disk data (table 1). They are closely correlated with "the network" (clumps) of L $\alpha$ emission.
} 
concentrations to CI regions, but $\mathrm{L} \alpha$ is bright also directly over the flux concentrations themselves.

In fact, $\mathrm{L} \alpha$ is brightest where loops much longer than $10 \mathrm{Mm}$ originate in the photosphere (lower panel of the figure). This is because there simply is not room for short loops to reach the central, brightest and unipolar regions of the flux concentrations from neighboring CI fields. These longer and taller loops, if they contain much plasma, are likely to have most of their volume filled with hot (i.e. coronal) plasma in order that they have a reasonable lifetime (i.e. close to hydrostatic equilibrium, e.g. Rosner et al. 1978). Thus, La emission from the NBs appears to be dominated by processes near the footpoints of long loops. The longer loops not only originate in the bright cores of the concentrations, but they also show "comet"-like structure in broad agreement with the L $\alpha$ thread morphology. These field line shapes arise because surface field lines initially directed towards the SE are forced to avoid the strong same polarity flux concentration there, and so turn towards the NW, attracted by opposite polarities outside of the FOV.

These results present difficulties for the "cool loop" model, which attempts to explain most of the network emission. However, care is needed before arriving at stronger conclusions. At the sensitivity of the KPVT data, there are simply too few small loops to account for all threaded L $\alpha$ structures. It may be that, as suggested by figure 1, there are unresolved mixed polarities within the cores of the flux concentrations, as well as in CI regions. To address this problem, we turn to more sensitive measurements of surface magnetic fields, at higher angular resolution, to see if such fields exist on the Sun at a level undetected by these data, and discuss the role of fields unresolved even by Hinode later.

\section{Vector polarimetry from Hinode}

\subsection{Longitudinal fields}

We examined data for quiet and active regions obtained with the SP on Hinode. Such data are unique, stable, seeing-free measurements of the full Stokes vector, with pixels of only $0.164^{\prime \prime}$. Image stability is critical for accurate polarimetry since seeing-induced errors can be large (Lites 1987), even for ground-based images captured with adaptive optics (Judge et al. 2004). The noise in longitudinal magnetograms from a "normal map" (4.8 second acquisition time) acquired with the SP corresponds to just $3 \mathrm{Mx} \mathrm{cm}^{-2}$ in each pixel (Lites et al. 2008). These data are sensitive to very small magnetic flux concentrations, the noise levels are $4 \times 10^{14} \mathrm{Mx}$ (Table 1$)$.

Active region data from December 292006 and January 192007 were analyzed with quiet 
data obtained on November 27 2007. Here we address the quiet (figure 6) and December 2006 (figure 7) datasets. Since the SP is a slit instrument, the regions took 135 and 43 minutes to scan respectively. The latter small active region contains a small group of pores and a single pore of opposite positive polarity. These pores have associated with them magnetically disturbed granulation. Figure 8 shows close-up views of these magnetic concentrations, again plotting groups of representative field lines whose lengths are less than and greater than 10 $\mathrm{Mm}$, and which have flux densities in each pixel greater than $6 \mathrm{Mx} \mathrm{cm}^{-2}$ (i.e. $2 \sigma$ above the noise). When observed at high magnetic sensitivities and spatial resolution, the quiet Sun and active regions have network-like flux concentrations which are surrounded by mixed polarity fields. The SP data show significant signal almost everywhere (there is no obvious "white noise" component in the spatial power spectra). The SP thus has not yet reached a limiting small scale of the photospheric magnetic structure.

By binning these Hinode data to the pixel size and sensitivity of the KPVT, we find that, as expected, the KPVT misses magnetic flux. But the missing flux amounts to just 25\% of the detectable flux within the supergranular CIs. Thus, although a significant number of short loops connecting NBs to CIs are certainly missing from figures 4 and 5 , the missing flux would not be sufficient to account for all observed $\mathrm{L} \alpha$ threads as cool loops.

NB fields are organized into concentrations of various sizes, so care must be taken in discussing field line lengths and their relation to underlying flux and associated UV emission. It is convenient to discuss two groups- those unipolar concentrations in excess of $5 \times 10^{18}$ $\mathrm{Mx}$, and those below.

The VAULT-2 and active region SP data fall into the large flux group, the quiet Sun SP data into the small flux group. In the large flux group, the SP data contain no examples of small scale $\left(\lesssim 10^{18} \mathrm{Mx}\right)$ mixed polarities within the cores of the network concentrations themselves, at the detection limit of $\sim 0.4 \times 10^{15} \mathrm{Mx}$, as is suggested by figure 1 . If the cool loop picture is generally applicable, there is a point where we should observe them. But the SP data show that, at a resolution of $240 \mathrm{~km}$, the typical large concentration is unipolar. Any missing mixed polarity fields on scales below $240 \mathrm{~km}$ would yield extremely short loops (lengths $\ell \lesssim 240 \pi / 2 \approx 365 \mathrm{~km}$ ) which would not even be visible in features like L $\alpha$ formed higher above the photosphere (see section 5.2). The SP data therefore confirm that short loops from larger concentrations associated with active network (an example might be that at $x=15$ in the lower panel of figure 8) generally only extend from the peripheries of these flux concentrations. The core regions of the concentrations are almost always connected via longer loops between different concentrations and not between the concentration and CI field (note the absence of short loops connected to centers of large concentrations of flux in the lower panel of figure 8). The brightest UV and EUV emission from the TR sits (statistically) 
directly over the concentrations, as exemplified by the VAULT-2 data above. The cool loop picture therefore fails to account for the bulk of L $\alpha$ emission over such NBs.

The story is different in the small flux group. The SP data contain examples of what are probably mixed polarities within NBs, as proposed by Dowdy et al. (1986). For example, if the negative polarity ribbon of condensations along $x=5$ are considered part of the larger positive polarity condensations seen along $x=7.5$ in the upper panel of figure 8 , then this situation qualifies as a mixed polarity network structure as suggested by Dowdy et al.. The short loops clustered between and around these concentrations are likely candidates for cool loops, and may explain the rosette like structures seen in the TR in the quiet Sun (see the compilation of images from the SUMER instrument on SOHO Feldman et al. 2003).

\subsection{Vector fields}

The SP data consist of the full Stokes vectors of the photospheric Fe I 6301.5 and $6302.5 \AA$ lines. We can therefore assess how the potential fields compare to the transverse field properties derived from the Stokes $Q, U$ (linear polarization) measurements. This is meaningful only for the areas of active region data with sufficient signal-to-noise ratios. Figure 9 shows the field azimuths both measured and computed using the MERLIN ${ }^{5}$ inversion scheme for the 29 December 2006 dataset. (Field azimuths are the angles measured counterclockwise from the direction pointing solar west, of the field vector projected on to the local solar surface. The azimuths are subject to the well known $180^{\circ}$ ambiguity). There is broad agreement. Significant departures from potential fields exist in both active region datasets, some arising simply from the limits of using Fourier transforms which assume that the domain is periodic in $x$ and $y$. (The "cusps" seen near $y=47$ in figure 7 are such artifacts, and the azimuths close to the boundaries in Figure 9 also reflect this problem). Others are of solar origin, caused by electrical currents above the surface $z=0$. Examples are the many patches of gray near the center of the lower panel of in Figure 9. These differences are of prime interest for the physics of the atmosphere, but here we ask simply how our conclusions concerning the validity of the "cool loop" model might be changed.

The lack of short loops in the cores of larger network flux concentrations appears to be a robust result- opposite polarities simply do not exist there for any length of time. The directions and connections of field lines will depart from the potential calculations. We do not speculate on such effects, simply noting the broad agreement in the measured and computed azimuths. But it is difficult to see how the "comet" structures could remain aligned as they

\footnotetext{
${ }^{5}$ http://www.hao.ucar.edu/projects/csac/nextgen.php\#merlin. See Lites et al. (2007).
} 
are seen in the VAULT-2 data by small-scale current systems. Indeed, it is well known that filaments must carry significant electrical currents to provide support against gravity via the Lorentz force (e.g. Low 1996). "Comet"-like structures seen in upper chromospheric H $\alpha$ are aligned on scales larger than supergranulation along filament channels (Martin et al. 1994). The large scale currents associated with filament channels may therefore also be responsible for the observed $\mathrm{L} \alpha$ "comet" alignment. As in the potential field case, it is only if the "comets" are associated with large scale (> supergranules) coronal structure that this observation makes physical sense.

\section{Discussion and conclusions}

On the basis of cool loop models of the VAULT-2 L $\alpha$ emission analyzed here, Patsourakos et al. (2007) concluded that " The reasonable agreement between the models and the observations indicates that an explanation of the observed fine structure in terms of cool loops is plausible." Their motivation for interpretation of the threads in terms of such models is that the thread-like structure, assumed to be aligned with magnetic fields associated with the chromospheric network, is incompatible with heating via field-aligned heat conduction down from overlying coronal plasma. The cool loop model has emerged as a viable explanation for the anomalous brightness of TR emission in features formed below $2 \times 10^{5} \mathrm{~K}$, in spite of some significant physical problems, notably the tendency for instability of such classes of model (Cally and Robb 1991). Nevertheless, the work by Patsourakos et al. (2007) lends support to this picture.

In contrast, by studying VAULT-2 data in terms of the magnetic structure of the chromospheric network, we find:

- The location and orientation of some of the $\mathrm{L} \alpha$ threads are only rarely compatible with the idea of cool, short, loops originating from the NBs and extending into neighboring CI regions, but

- such short loops usually connect the CI to the peripheries of network flux concentrations with unipolar fluxes in excess of, say $5 \times 10^{18} \mathrm{Mx}$.

- The bulk of the L $\alpha$ NB emission, arising from the cores of such concentrations, seems to be associated with far longer magnetic loops which connect to other concentrations. In this way these concentrations seem analogous to plages.

- In the "quiet" region of the VAULT-2 data, the longer loops diverge non-radially from their NB concentrations in a manner reminiscent of the "comet" L $\alpha$ patterns. 
- Hinode SP observations at 0"33 resolution reveal that short loops can exist in what appear to be NBs, provided the concentrations making up the magnetic concentrations have small enough areas (fluxes $\lesssim 5 \times 10^{18} \mathrm{Mx}$ ). Thus, cool loops emitting L $\alpha$ may indeed be present in quiet regions, where they may cluster around small concentrations of flux, and have the appearance of clumped rosettes of emission of $\lesssim 10 \mathrm{Mm}$ diameter (see the upper panel of Figure 8).

- Hinode data in the neighborhood of active regions, while showing mixed polarities, tend to reveal short loops only connecting peripheries of the larger flux concentrations found there to the CI regions (see the lower panel of Figure 8). This is also the case found for the particular VAULT-2 data analyzed here. The bulk of bright network L $\alpha$ emission is difficult to accounted for by such structures.

- Non-potential fields are clearly present in the Hinode data, but we have argued that such fields, on the small scales associated with the cool loop model, do not affect our overall conclusions.

Our work suggests that the cool loop picture cannot be universally valid. Below we propose a different qualitative picture of the emission from the cores of these flux concentrations.

\subsection{The "comet" patterns seen in VAULT data}

The aligned "comet-tail" patterns of the L $\alpha$ threads initially presented us with a puzzle. Why would such large scale order be characteristic of a small scale process involving the formation of physically far smaller loops, whose footpoints might be controlled by random convective processes?

The explanation may be that the region termed "quiet" by Patsourakos and colleagues is not really "quiet". There is close by a large net flux which imposes a large scale order on potential fields. Two other observations indicate organization on a large scale: the threads largely point away from the coronal Fe IX/X emitting loops associated with the active region in the SE corner of the field of view; the "quiet" region in fact lies between two filament channels- the SE channel and another lying along the western edge of the VAULT FOV (see fig. 2). Free magnetic energy associated with the magnetostatic balance of filaments, in the form of atmospheric current systems, is surely present which might modify the overlying magnetic field from the potential state we have calculated. Such currents would not be incompatible with the large scale order implied by the comets. It is only if one considers 
the structures to be formed from convection-driven short loops that large scale organization would be surprising. Our analysis instead points to an association of long loops with most of the L $\alpha$ emission, including the threads modeled previously as cool loops. Instead of cool loops, the emission seems to arise, perhaps as chromospheric material is launched as spicules and heated along much longer field lines. The difficulty in this picture is to explain why $\mathrm{L} \alpha$ emission is bright and extended over several Mm lengths, given the obvious failure of field-aligned heat conduction to achieve this.

\subsection{The role of unresolved magnetic fields}

We noted above that the SP data show signal on all scales down to the Lunqvist limit (2 pixels $=0$.'328). The smallest scales of solar photospheric magnetic fields are as yet unknown. It is therefore likely that tiny loop structures are missing from our analysis. However, the existence of smaller scale structures does not weaken our conclusions, for several reasons.

Firstly, to the extent that the magnetic fields are potential, structures of horizontal scale $\ell$ in the photospheric normal magnetic field will extend only to heights $\approx \ell$ higher into the atmosphere. This result is a general property of solutions of Laplace's equation (see, e.g. Gary 1989). Now L $\alpha$ radiation cannot emerge from heights less than the height where the continuum optical depth is unity, which occurs near $0.8 \mathrm{Mm}$ because of opacity and atmospheric stratification (FAL). In FAL's models, the bulk of the emission arises at least $2 \mathrm{Mm}$ above the photosphere. Thus, for any small-scale bipoles observable at the solar surface, only those with footpoints separated by $0.8 \mathrm{Mm}$ or so can contribute to observable L $\alpha$ emission. Those separated by smaller scale lengths likely return to the photosphere before reaching $1 \mathrm{Mm}$ heights. The thermal signatures of such loops would be primarily visible in lines and continua less opaque than $\mathrm{L} \alpha$, influencing $\mathrm{L} \alpha$ itself only marginally.

Other reasons arise from the nature of the observed $L \alpha$ threads. They extend over $5-10 \mathrm{Mm}$ lengths, so that if formed within tubes of magnetic flux, footpoint separations are at least this large. Threads of lengths between 2 and $5 \mathrm{Mm}$ would extend high enough to emit $\mathrm{L} \alpha$ but are not seen in the data. The thread orientations are collectively organized over scales of several supergranules, and the threads, if both footpoints are anchored in the photosphere, require opposite polarity flux surrounding the flux concentrations associated with network boundaries. None of these observations are consistent with random "salt and pepper" distributions of flux associated with supergranules on any scale below say $5 \mathrm{Mm}$ in length.

For all these reasons we believe that unobservably small scale magnetic fields are irrele- 
vant to the problem of understanding the essential properties of the observed L $\alpha$ line, both in plage and other regions.

\subsection{Speculation on the origin of the bulk of NB emission}

Field-aligned diffusive and flowing models of the type computed by FAL may indeed account for the moss $\mathrm{L} \alpha$ emission, but as noted in the introduction, they are incompatible with $\mathrm{L} \alpha$ thread emission. In the absence of cool loops as a viable proposal of $\mathrm{L} \alpha$ emission except in quiet regions, we can speculate on what might be the cause of the L $\alpha$ properties seen by VAULT.

Figure 3 reveals that the corona overlying the observed region is bright on the SE side, dim elsewhere. The L $\alpha$ emission from network patches seems to care little of the intensity of overlying coronal emission. If energy for $\mathrm{L} \alpha$ radiation arises directly from coronal plasma, surely there must be some correlation between L $\alpha$ and coronal brightness?

Independent evidence suggests that the observed L $\alpha$ structure is correlated with observations of $\mathrm{H} \alpha$, whose morphology is complicated, but whose properties on fine scales are known to relate to conditions in the overlying corona (Berger et al. 1999). Berger et al. (1999) found that, on scales of arcseconds and less, coronal "moss" emission at $171 \AA$ is dark where $\mathrm{H} \alpha$ wings are strong, suggesting that the EUV corona and cooler $\mathrm{H} \alpha$ plasma are separated by a thermal interface which lies parallel to magnetic field lines (it is not therefore the "classical" thin TR, but more of a sheath.) We can speculate that this interface might by a place where energy from the coronal plasma can be transferred to hydrogen atoms, by diffusion of neutral atoms into the hot coronal regions (Pietarila and Judge 2004), via cross-field conduction which occurs because of proton dynamics (Athay 1990), or because of some as yet undetermined (Rayleigh-Taylor like?) instability (Gabriel 1976).

Given this interface, consider the neutral atom diffusion scenario (Judge 2008, submitted). Neutral atoms, at an interface with hot corona, experience no Lorentz force until ionized. The probability that a hydrogen atom is ionized by collisions with hot coronal particles (electrons) is related to the probability for excitation to the $n=2$ level, which is almost immediately $\left(10^{-8} \mathrm{~s}\right)$ followed by the emission of a $\mathrm{L} \alpha$ photon. Roughly one $\mathrm{L} \alpha$ photon is emitted before it is ionized, independent of the density of the coronal plasma, provided it is ionized somewhere in the coronal plasma. It turns out that the L $\alpha$ intensities expected from this process are proportional to the thermal energy density of the corona and the neutral diffusion speed. Thus, there is a different dependence of coronal and L $\alpha$ emission on thermal parameters, so it is possible in principle to explain why similar L $\alpha$ intensities may 
arise from regions with different coronal intensities. The essential difference between this and the cool loop picture is that coronal thermal energy is drained by diffusion of neutrals across magnetic field lines to generate much of the emission seen in L $\alpha$ and other typical "lower TR transitions". Some chromospheric process is assumed to launch spicules to get the process started. Such a model can explain the puzzle of the "comet" asymmetry noted in the present paper, in that cool plasma threads along long coronal loops already have the large-scale organization required by observations of the $L \alpha$ threads. If it proves feasible, this process bypasses thermal stability problems presented by cool-loop models, and it is appealing in that the downward directed conductive flux density of $\approx 10^{6} \mathrm{ergs} \mathrm{cm}^{-2} \mathrm{~s}^{-1}$, unaccounted for in cool loop models, is radiated by cool, strong TR lines. Further work on this scenario is in progress (Judge, 2008, submitted).

We thank Scott McIntosh and the referee for helpful comments on this paper. Hinode is a Japanese mission developed and launched by ISAS/JAXA, collaborating with NAOJ as a domestic partner, NASA and STFC (UK) as international partners. Scientific operation of the Hinode mission is conducted by the Hinode science team organized at ISAS/JAXA. This team mainly consists of scientists from institutes in the partner countries. Support for the post-launch operation is provided by JAXA and NAOJ (Japan), STFC (U.K.), NASA, ESA, and NSC (Norway).

\section{REFERENCES}

Allen, C. W.: 1973, Astrophysical Quantities, Athlone Press, Univ. London

Anderson-Huang, L. S.: 1998, Space Sci. Rev. 85, 203

Antiochos, S. K. and Noci, G.: 1986, Astrophys. J. 301, 440

Ashbourn, J. M. A. and Woods, L. C.: 2001, Proc. R. Soc. A. 457(2012), 1873

Athay, R.: 1990, Astrophys. J. 362, 364

Athay, R. G.: 1981, Astrophys. J. 249, 340

Berger, T. E., De Pontieu, B., Schrijver, C. J., and Title, A. M.: 1999, Astrophys. J. Lett. 519, L97

Cally, P. S.: 1990, Astrophys. J. 355, 693

Cally, P. S. and Robb, T. D.: 1991, Astrophys. J. 372, 329 
Delaboudiniere, J.-P., and 27 co-authors: 1995, Solar Phys. 162, 291

Di Giorgio, S., Reale, F., and Peres, G.: 2003, Astron. Astrophys. 406, 323

Dowdy, J. F., J., Rabin, D., and Moore, R. L.: 1986, Solar Phys. 105, 35

Feldman, U.: 1983, Astrophys. J. 275, 367

Feldman, U., Dammasch, I. E., Wilhelm, K., Lemaire, P., Hassler, D. M., and Battrick, B.: 2003, Images of the solar upper atmosphere from SUMER on SOHO, ESA SP-1274. Noordwijk, Netherlands: ESA Publications Division

Fletcher, L. and de Pontieu, B.: 1999, Astrophys. J. Lett. 520, L135

Fontenla, J. M., Avrett, E. H., and Loeser, R.: 1990, Astrophys. J. 355, 700

Fontenla, J. M., Avrett, E. H., and Loeser, R.: 1991, Astrophys. J. 377, 712

Fontenla, J. M., Avrett, E. H., and Loeser, R.: 1993, Astrophys. J. 406, 319

Fontenla, J. M., Avrett, E. H., and Loeser, R.: 2002, Astrophys. J. 572, 636

Gabriel, A.: 1976, Phil Trans. Royal Soc. Lond. 281, 339

Gary, G. A.: 1989, Astrophys. J. Suppl. Ser. 69, 323

Hammerschlag, R. H. and Bettonvil, F. C. M.: 1998, New Astronomy Reviews 42, 485

Handy, B. N., and 47 co-authors: 1999, Solar Phys. 187, 229

Jordan, C.: 1980, Astron. Astrophys. 86, 355

Judge, P. G., Elmore, D. F., Lites, B. W., Keller, C. U., and Rimmele, T.: 2004, Applied Optics: optical technology and medical optics submitted

Kiepenheuer, K. O.: 1953, in G. P. Kuiper (Ed.), The Sun, Chicago University Press, Chicago, p. 322

Korendyke, C. M., Vourlidas, A., Cook, J. W., Dere, K. P., Howard, R. A., Morrill, J. S., Moses, J. D., Moulton, N. E., and Socker, D. G.: 2001, Solar Phys. 200, 63

Kosugi, T., and 24 co-authors: 2007, Solar Phys. 243, 3

Lites, B., Casini, R., Garcia, J., and Socas-Navarro, H.: 2007, Memorie della Societa Astronomica Italiana 78, 148 
Lites, B. W.: 1987, Applied Optics 26, 3838

Lites, B. W., Elmore, D. F., and Streander, K. V.: 2001, in M. Sigwarth (Ed.), Advanced Solar Polarimetry - Theory, Observation, and Instrumentation, Vol. 236 of Astronomical Society of the Pacific Conference Series, 33

Lites, B. W., Kubo, M., Socas-Navarro, H., Berger, T., Frank, Z., Shine, R., Tarbell, T., Title, A., Ichmoto, K., Katsukawa, Y., Tsuneta, S., Sumematsu, Y., Shimizu, T., and Nagata, S.: 2008, Astrophys. J. 672, 1237

Livingston, W. and Harvey, J.: 1971, in R. Howard (Ed.), Solar Magnetic Fields, IAU Symposium 43, Reidel, Dordrecht, p. 51

Low, B. C.: 1996, Solar Phys. 167, 217

Mariska, J. T.: 1992, The Solar Transition Region, Cambridge Univ. Press, Cambridge UK

Martin, S. F., Bilimoria, R., and Tracadas, P. W.: 1994, in R. J. Rutten and C. J. Schrijver (Eds.), Solar Surface Magnetism, 303

Patsourakos, S., Gouttebroze, P., and Vourlidas, A.: 2007, Astrophys. J. 664, 1214

Pietarila, A. and Judge, P. G.: 2004, Astrophys. J. 606, 1239

Pneuman, G. and Kopp, R.: 1978, Sol. Phys. 57, 49

Rabin, D. and Moore, R.: 1984, Astrophys. J. 285, 359

Reeves, E. M.: 1976, Solar Phys. 46, 53

Rosner, R., Tucker, W. H., and Vaiana, G. S.: 1978, Astrophys. J. 220, 643

Roumeliotis, G.: 1991, ApJ 379, 392

Sánchez Almeida, J., Teriaca, L., Sütterlin, P., Spadaro, D., Schühle, U., and Rutten, R. J.: 2007, Astron. Astrophys. 475, 1101

Scherrer, P. H., Bogart, R. S., Bush, R. I., Hoeksema, J. T., Kosovichev, A. G., Schou, J., Rosenberg, W., Springer, L., Tarbell, T. D., Title, A., Wolfson, C. J., Zayer, I., and MDI Engineering Team: 1995, Solar Phys. 162, 129

Skumanich, A., Smythe, C., and Frazier, E. N.: 1975, Astrophys. J. 200, 747

Tousey, R.: 1971, Royal Society of London Philosophical Transactions Series A 270, 59 
Vernazza, J., Avrett, E., and Loeser, R.: 1981, Astrophys. J. Suppl. Ser. 45, 635

Vourlidas, A., Klimchuk, J. A., Korendyke, C. M., Tarbell, T. D., and Handy, B. N.: 2001, Astrophys. J. 563, 374

Wilhelm, K., and 17 co-authors: 1995, proc. SPIE 2517, 2

Woods, D. T.: 1986, NCAR/CT 105

This preprint was prepared with the AAS LATEX macros v5.2. 
Table 1. Sensitivity of MDI, KPVT and Hinode-SP longitudinal magnetograms

\begin{tabular}{llll}
\hline \hline Instrument/mode & $\begin{array}{l}\text { noise per pixel } \\
\text { Mx cm }\end{array}$ & $\begin{array}{l}\text { pixel size } \\
\text { arc seconds }\end{array}$ & $\begin{array}{l}\text { noise in flux } \\
\text { units of } 10^{15} \mathrm{Mx}\end{array}$ \\
\hline MDI/full disk & 17 & $1^{\prime \prime} .984 \times 1^{\prime \prime} .984$ & 350 \\
KPVT/synoptic & 2.8 & $1^{\prime \prime} 148 \times 1^{\prime \prime} 148$ & 19 \\
Hinode SP/normal map & 3 & $0^{\prime \prime} .164 \times 0^{\prime \prime} .164$ & 0.42 \\
(Kitt Peak 40 channel magnetograph & 0.4 & $\dagger$ & $\approx 13$ \\
Livingston and Harvey 1971) & & & \\
\hline
\end{tabular}

Note. - $1^{\prime \prime}$ on the Sun corresponds to $725 \mathrm{~km}$ (Allen 1973). †'Seeing limited, here we use an effective pixel size of $2.5 \times 2.5^{\prime \prime}$ corresponding to half of the quoted resolution of $5^{\prime \prime}$. 


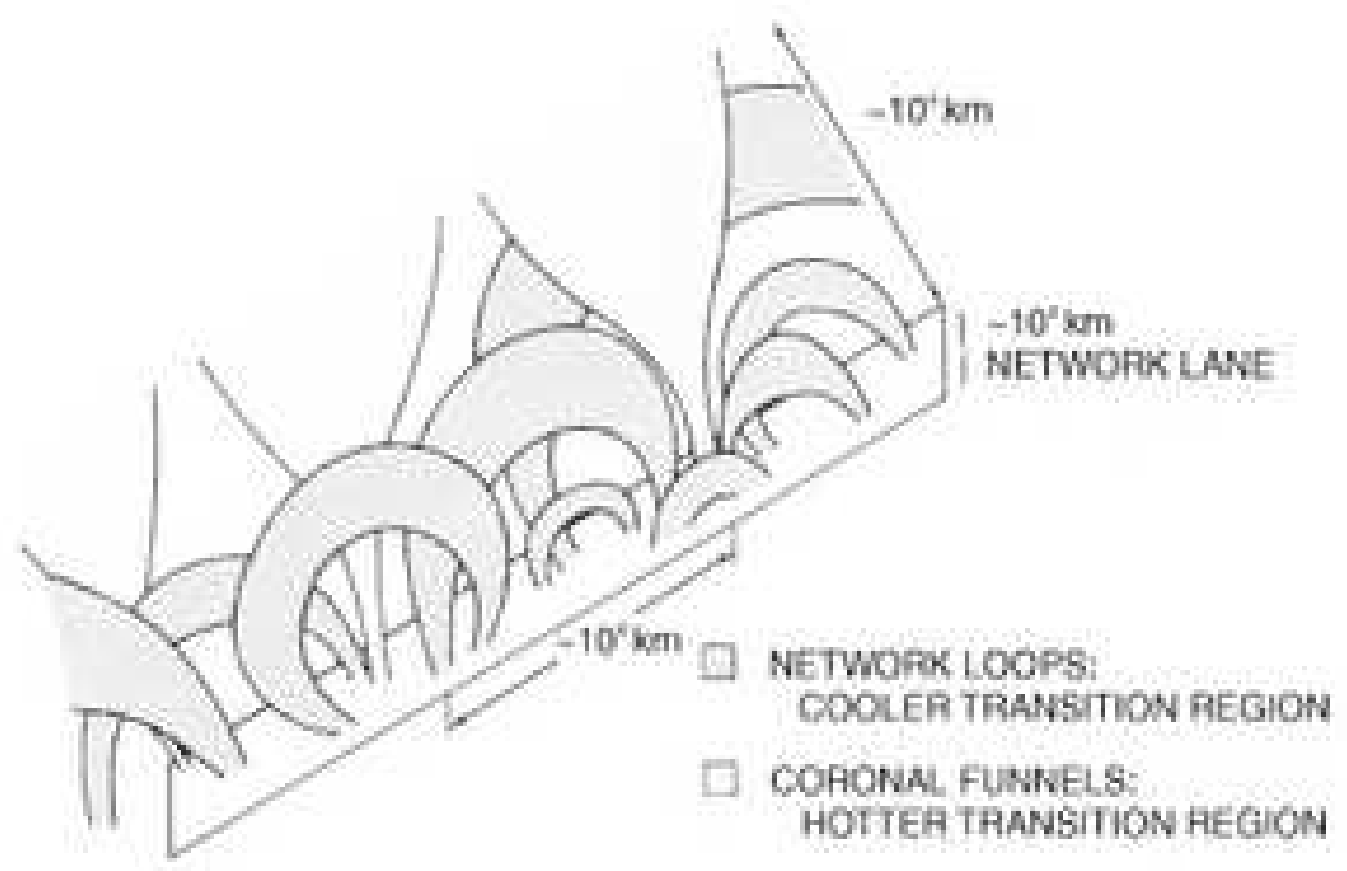

Fig. 1.- A sketch of the likely magnetic structure in a quiet region of the Sun's magnetic network, according to Dowdy et al. (1986). The "coronal funnels" are similar to the structures modeled by Gabriel (1976), which can account for emission above about $2 \times 10^{5} \mathrm{~K}$. The "network loops", arising from mixed polarity magnetic fields within network boundaries, explain the cool transition region emission, in Dowdy's picture. 

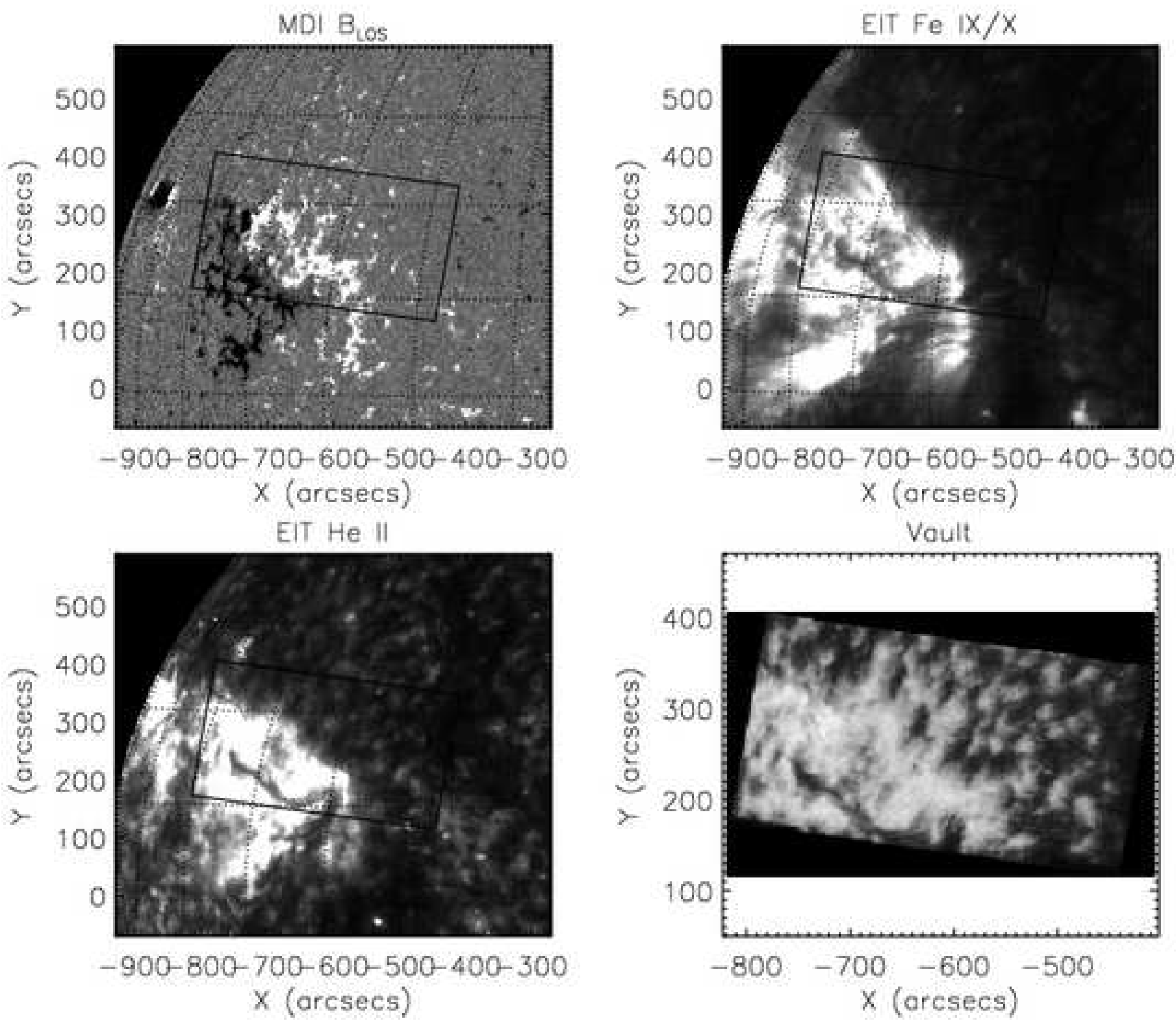

Fig. 2. - Context images showing the VAULT FOV (boxed region) for the observation at 18:12 UT on 14 June 2002. Shown are images of longitudinal field from MDI (between $\pm 200 \mathrm{Mx} \mathrm{cm}^{-2}$ ), and of He II 304 emission and $171 \AA$ Fe IX/X emission from EIT, both instruments on the SOHO spacecraft. These images were differentially rotated to 18:12:00 UT, the epoch of these VAULT-2 observations. The active region in the SE corner contains a filament along a magnetic neutral line, another filament is seen in the He II image, oriented roughly N-S on the western edge of the VAULT FOV. Also shown are (lower right) VAULT-2 data. 


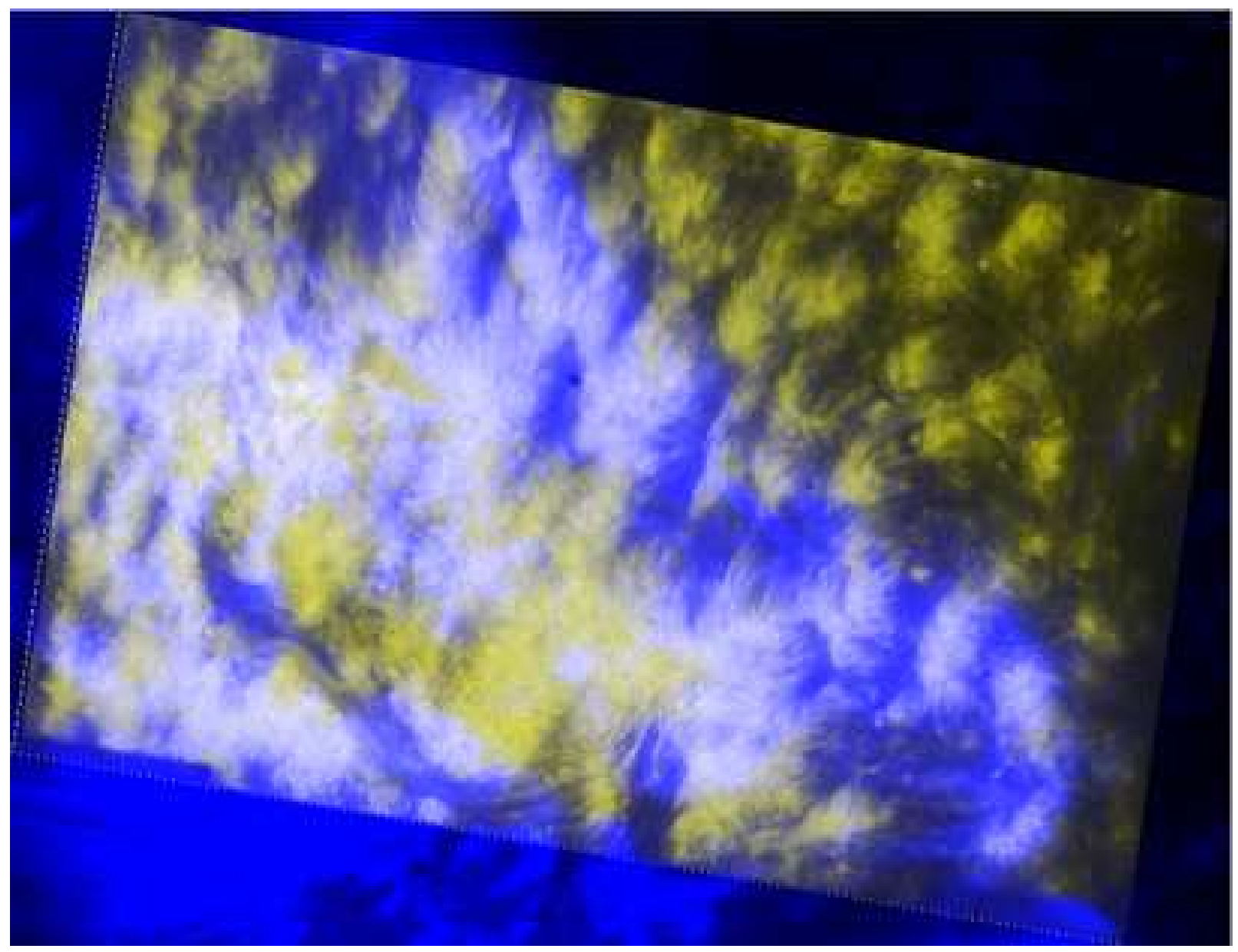

Fig. 3.- A false color image of VAULT-2 data (yellow) together with TRACE $171 \AA$ data of the lower corona (blue). The TRACE data were obtained 30 minutes before the VAULT-2 image. Four TRACE $171 \AA$ images were added together. 

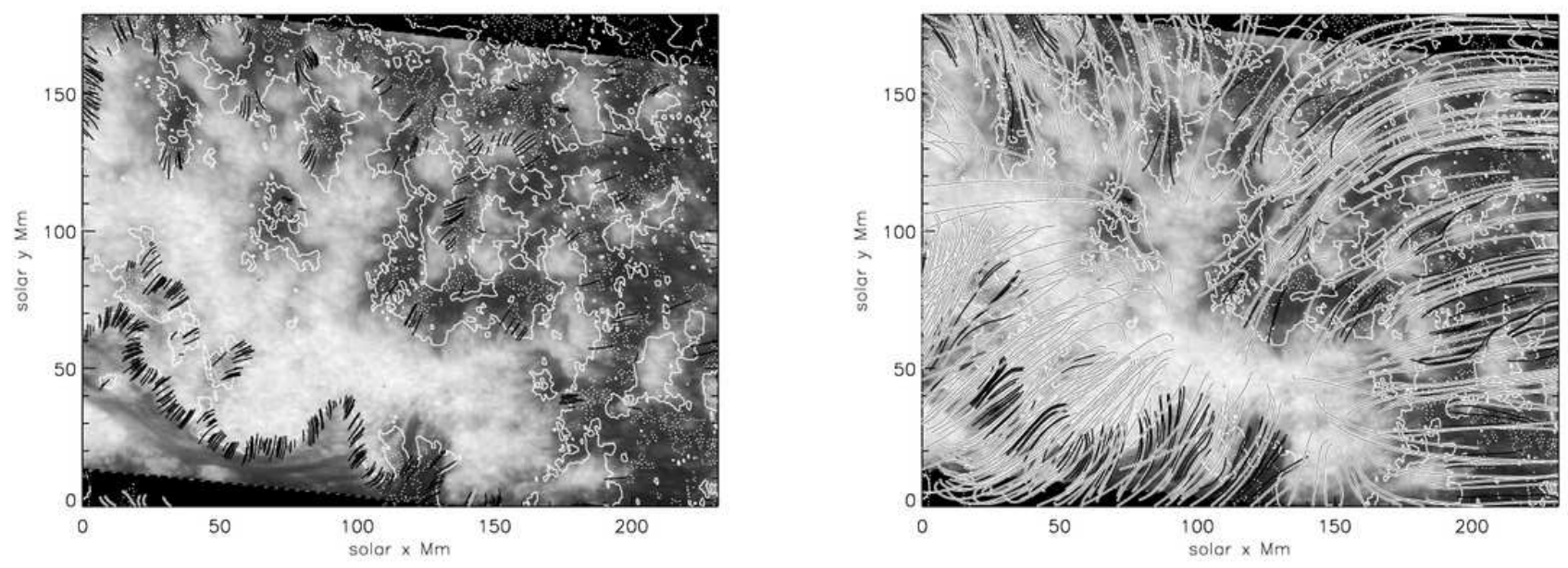

Fig. 4.- Magnetic field contours and potential field lines superposed on the VAULT-2 image. The upper panel shows loops with lengths $<10 \mathrm{Mm}$, the lower shows longer loops. Contours are at $\pm 2 \sigma\left( \pm 5.6 \mathrm{Mx} \mathrm{cm}^{-2}\right)$, negative contours are dashed lines, positive solid. Not all field lines from each pixel are plotted even if their signal exceeds the noise (see text), to avoid confusion. Field lines reaching heights $\leq 5 \mathrm{Mm}$ are plotted as a black line, others are shown as a black on top of a white line. The figure origins are the same but arbitrary, the center of the figure is Sun center. 

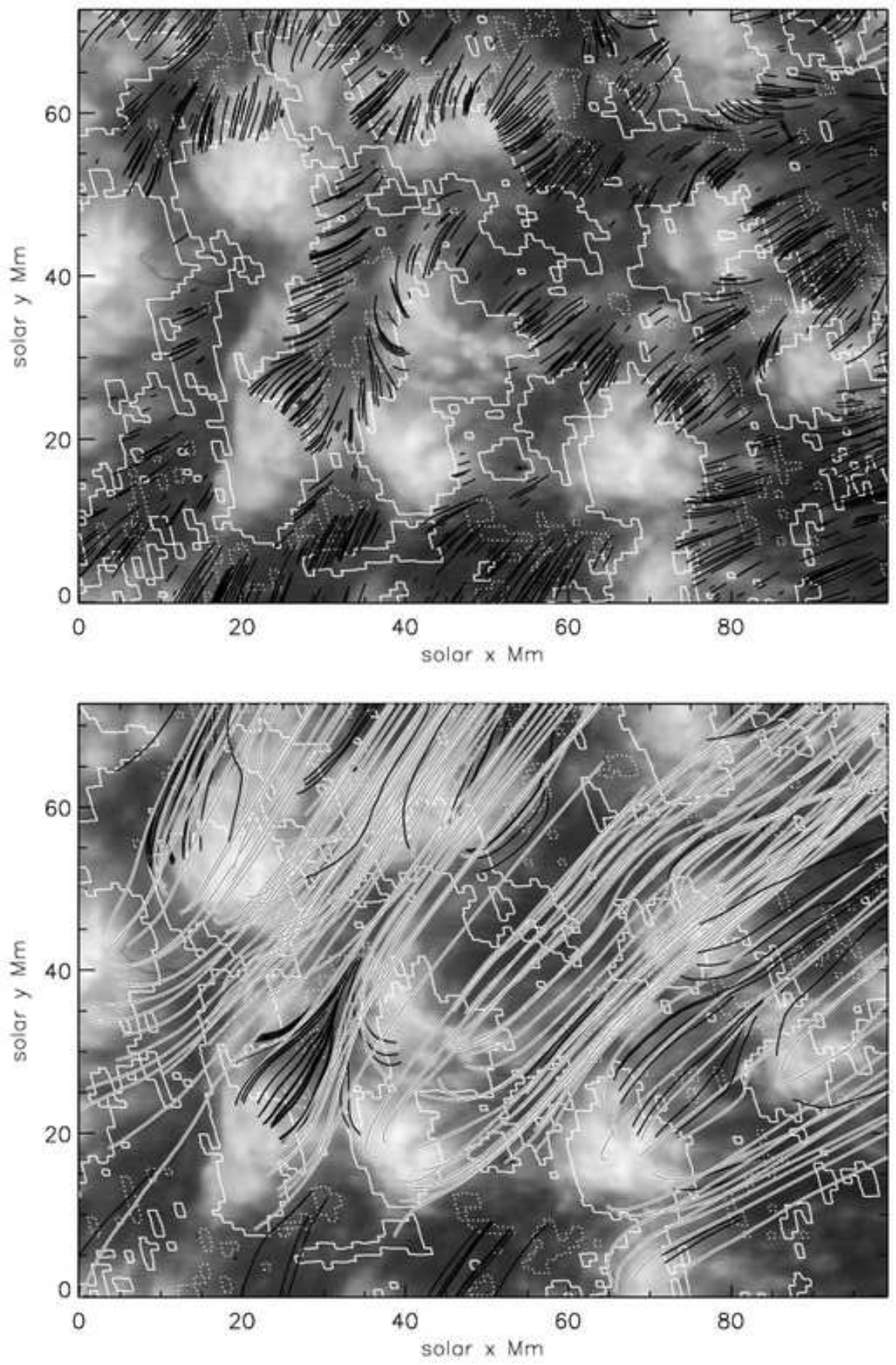

Fig. 5.- Potential field lines superposed on a portion of the VAULT-2 image, centered at $x, y=-500^{\prime \prime},+290^{\prime \prime}$ in Figure 2. The area covers perhaps 10 supergranular areas. The upper panel shows loops of $10 \mathrm{Mm}$ length and less, the lower panel longer loops. Contours of $\pm 2 \sigma$ $\left( \pm 5.6 \mathrm{Mx} \mathrm{cm}^{-2}\right)$ are shown, where $\sigma$ is the rms uncertainty in line of sight field strength. Field lines were plotted with no limit set on the signal-to-noise ratios of the magnetogram. There is little correspondence between short loops and the bright L $\alpha$ emission, instead the brightest emission originates from the bases of loops longer than $10 \mathrm{Mm}$. 

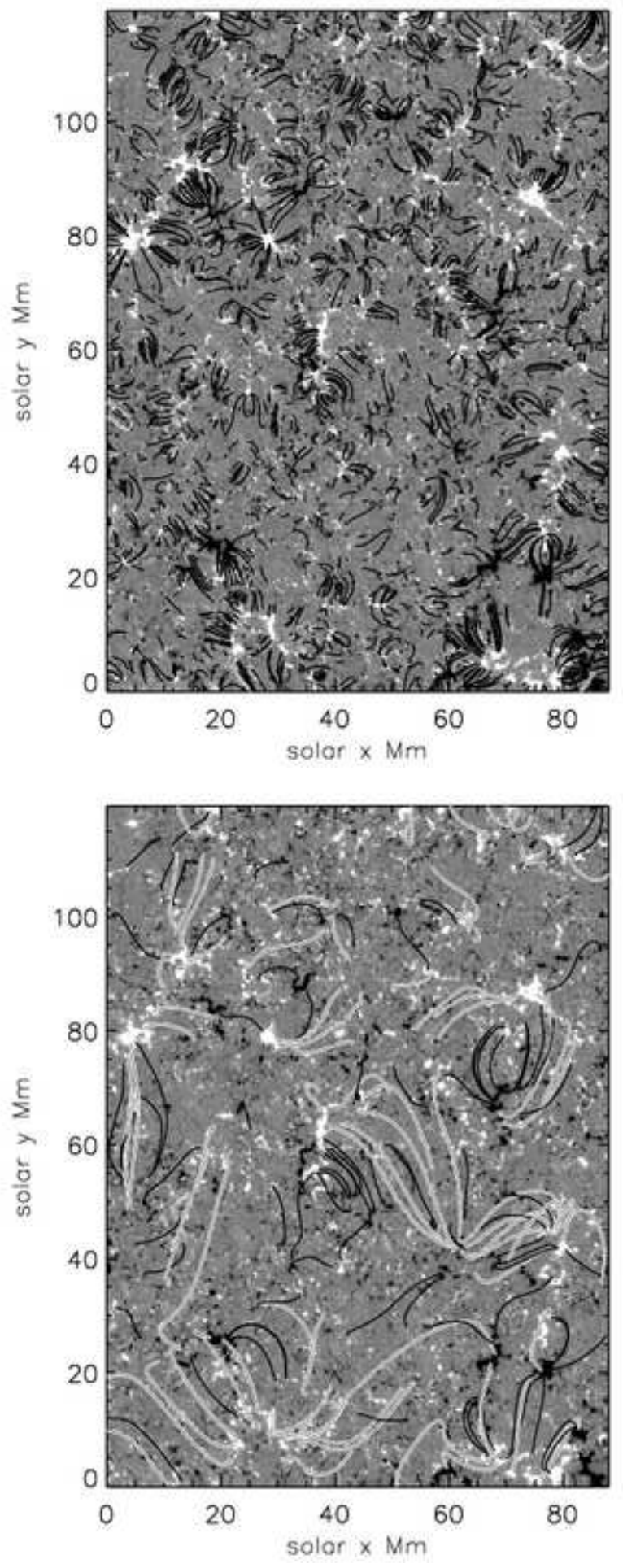

Fig. 6. - Line of sight field strength images, obtained with the SP on Hinode on 17 November 2007 beginning at 11:31 UT, are shown with potential field lines superposed. The magnetograms are shown on a linear scale between -50 and $+50 \mathrm{Mx} \mathrm{cm}^{-2}$ to show weak flux regions. The upper panel shows loops with total length below $10 \mathrm{Mm}$, the lower shows those with longer lengths. The coordinates have an arbitrary origin. 

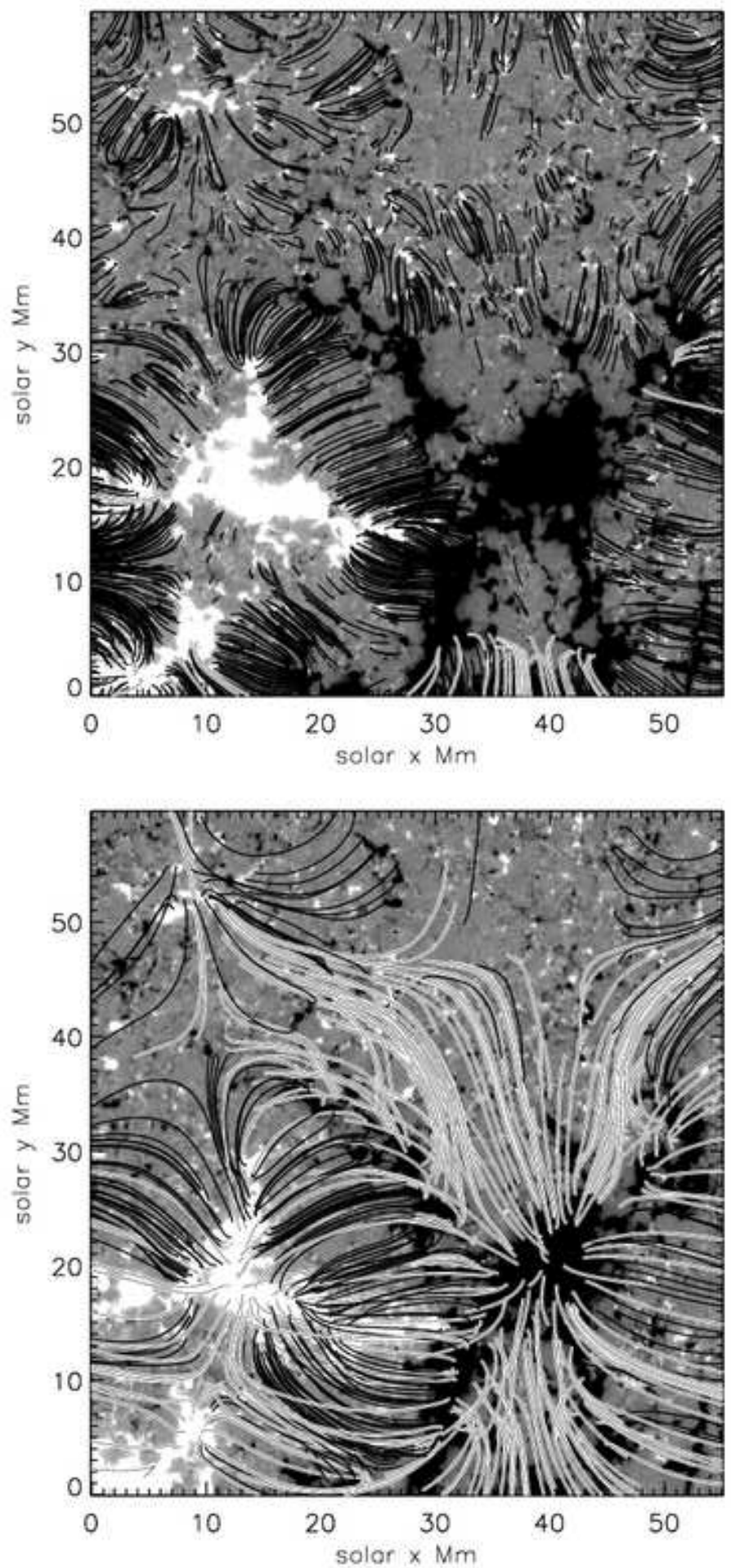

Fig. 7.- A similar plot to figure 6, but for the active region observed with the SP on Hinode, on 29 December 2006 beginning at 01:51 UT. (Field line cusps near $y=47$ are artifact of the Fourier method used.) 

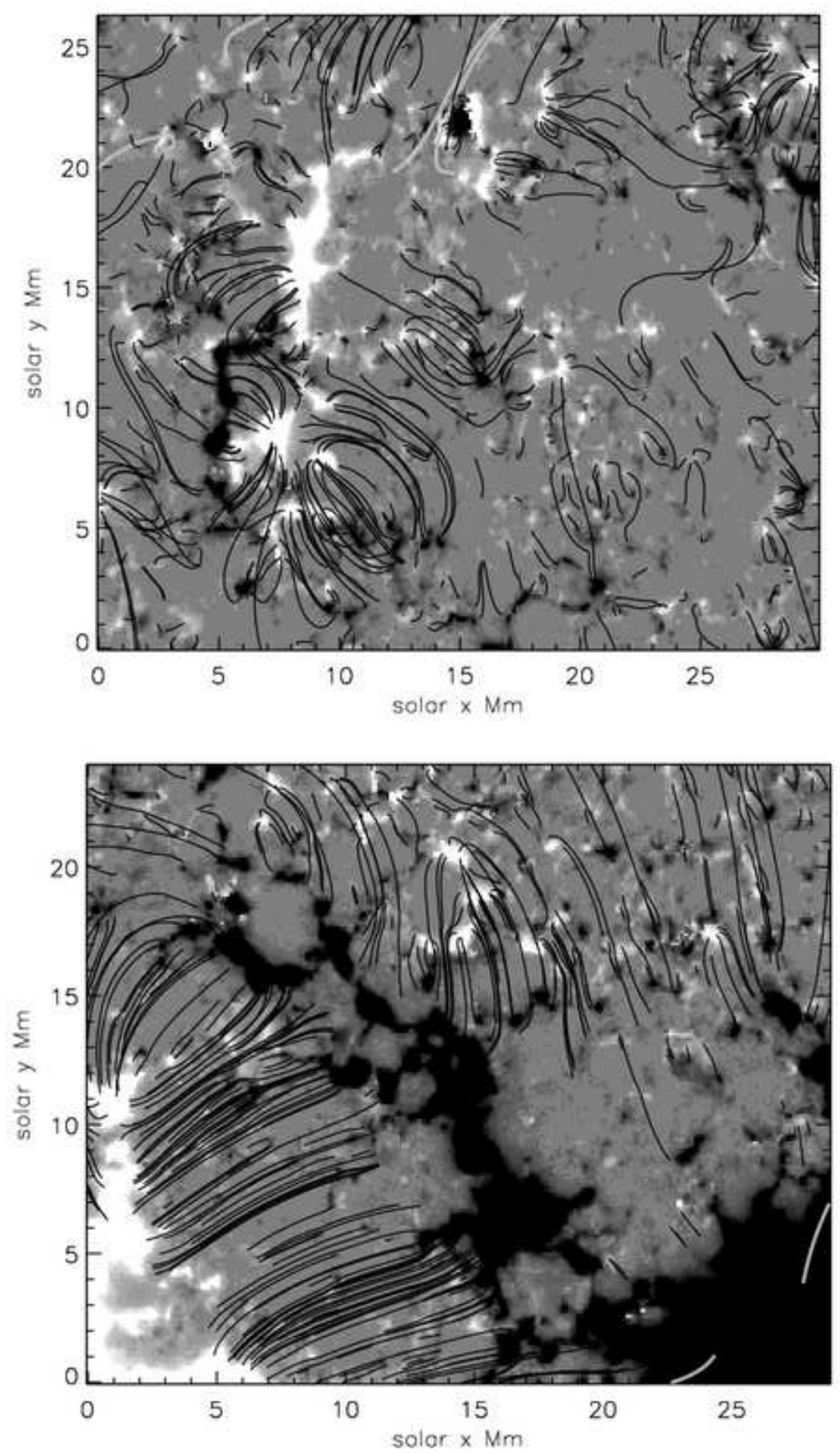

Fig. 8.- Close-up magnetograms of flux concentrations observed by the SP on Hinode from the central portions of the quiet region dataset from November 272007 (upper panel), and the more active area from and 29 December 2006 (lower panel). The regions show cover about the area of one supergranule. Short magnetic loops abound in both regions, as the strong network concentrations return to cell interiors of opposite polarity or to nearby concentrations. 

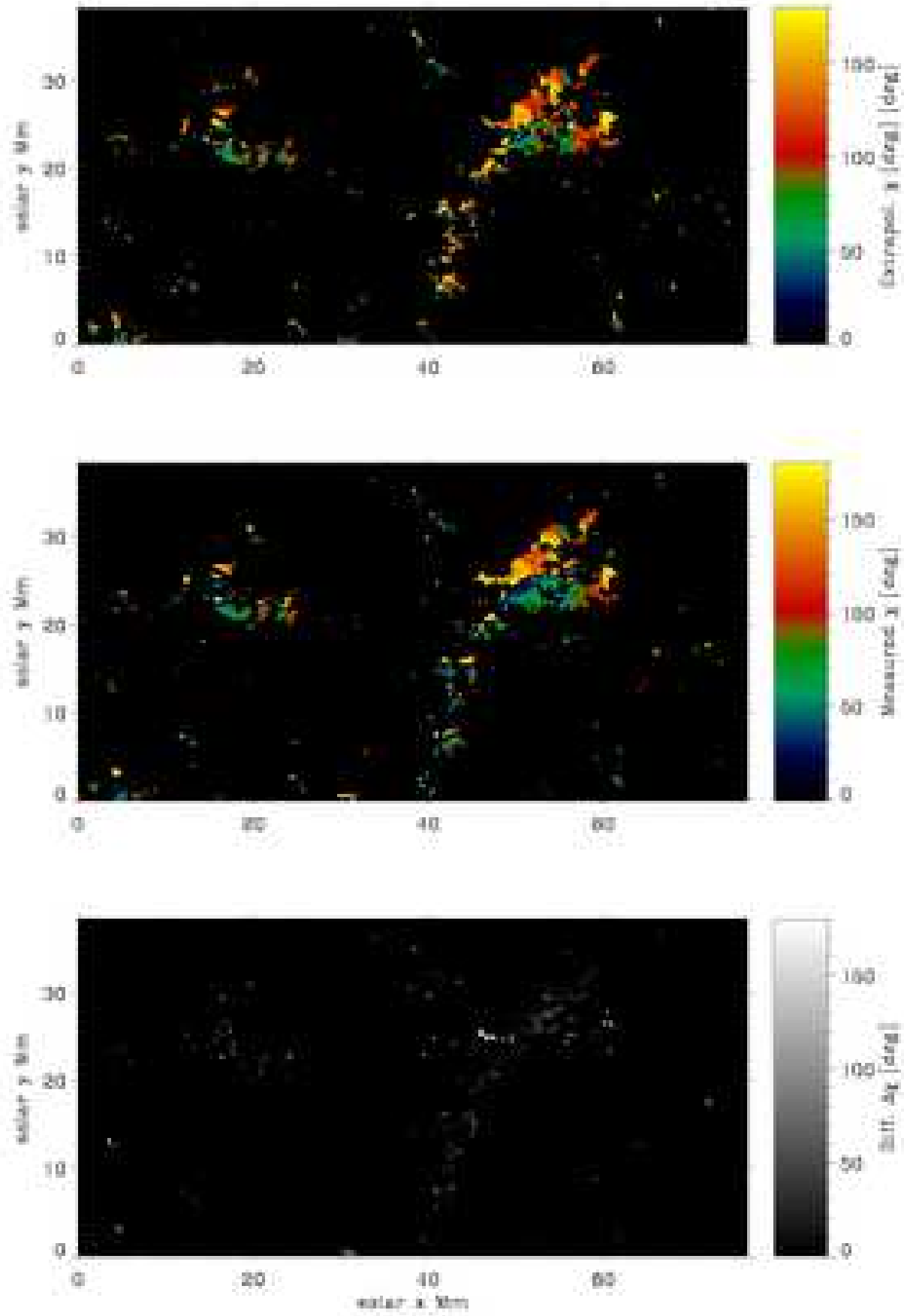

Fig. 9.- Magnetic field azimuths from inversions of the measured Hinode SP data (top), those computed from the potential field approximation using just the line-of-sight component of the magnetic field (center), and the difference (bottom). The plot shows the lower half of the dataset obtained on 29 December 2006 which contains significant Stokes $Q, U$ signals. White regions in the bottom panel are most likely the result of the $180^{\circ}$ ambiguity in inverted fields. The qualitative agreement suggests that such differences, if typical, do not affect our conclusions concerning the locations of loops of various lengths. 